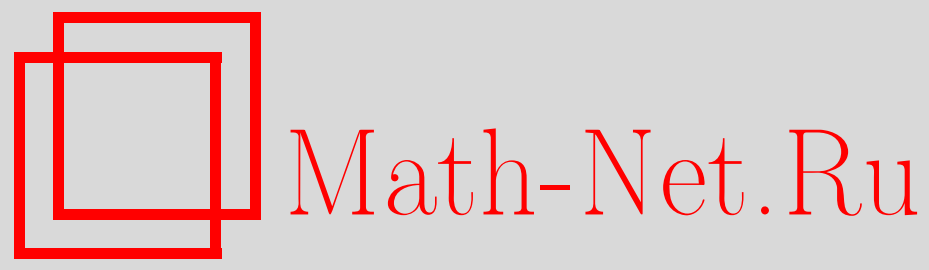

А. А. Джалилов, К. М. Ханин, Об инвариантной мере для гомеоморфизмов окружности с изломом, Функи. анализ и его прил., 1998, том 32, выпуск 3, 11-21

DOI: https://doi.org/10.4213/faa419

Использование Общероссийского математического портала MathNet.Ru подразумевает, что вы прочитали и согласны с пользовательским соглашением http://www . mathnet.ru/rus/agreement

Параметры загрузки:

IP : 54.224 .187 .69

26 апреля 2023 г., 17:38:08

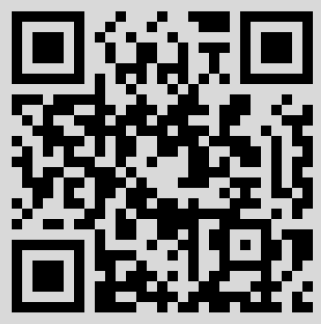




\title{
Об инвариантной мере для гомеоморфизмов окружности с изломом
}

\author{
(c) 1998. А. А. ДжАлИлов, К. М. ХАНИн
}

\section{§1. Введение}

В настоящей работе изучаются гомеоморфизмы окружности $S^{1}=[0,1)$ с одним изломом, т. е. со скачком первой производной в одной точке. Рассмотрим множество непрерывных строго монотонных функций $f(x), x \in \mathbb{R}^{1}$, со следующими свойствами:

(a) $0 \leqslant f(0)<1$;

(b) $f(x+1)=f(x)+1$;

(c) существует точка $x_{c} \in S^{1}$, такая, что в этой точке определены односторонние производные $f^{\prime}\left(x_{c}-0\right), f^{\prime}\left(x_{c}+0\right)>0$ и

$$
\sqrt{\frac{f^{\prime}\left(x_{c}-0\right)}{f^{\prime}\left(x_{c}+0\right)}}=c \neq 1 ;
$$

(d) $f(x) \in C^{2+\nu}\left(\left[x_{c}, x_{c}+1\right]\right), f^{\prime}(x)>0, x \in\left(x_{c}, x_{c}+1\right)$.

Всякий сохраняющий ориентацию гомеоморфизм окружности с одним изломом однозначно определяется функцией $f(x)$ и задается формулой

$$
T x=\{f(x)\}, \quad x \in S^{1},
$$

где $\{\cdot\}$ обозначает дробную часть числа. Обозначим через $\rho=\rho(T)$ число вращения, отвечающее гомеоморфизму $T$.

Если $\rho$ иррационально, то для достаточно гладких диффеоморфизмов траектория любой точки всюду плотна на окружности, а сам диффеоморфизм с помощью гомеоморфной замены координат приводится к повороту на угол $\rho$, т.е. существует гомеоморфизм окружности $\varphi$, такой, что

$$
\varphi(T x)=T_{\rho} \varphi(x), \quad x \in S^{1},
$$

где $T_{\rho} x=\{x+\rho\}$ - поворот окружности на угол $\rho$. Этот классический результат, доказанный Данжуа [1], без труда переносится на случай гомеоморфизмов с особенностью типа излома. Ниже приводится точная формулировка соответствуюшей теоремы.

Естественно возникает важный вопрос о гладкости сопрягающего гомеоморфизма $\varphi$. Первые результаты в этой области были получены В. И. Арнольдом [2] и Ю. Мозером [3] в рамках теории Колмогорова-Арнольда-Мозера (KAM). Впервые глобальный результат о гладкости $\varphi$ был доказан М. Эрманом $[4,5]$. В дальнейшем он был обобщен в работах Йокоза [6], Синая и Ханина $[7,8]$, Орнштейна и Катцнельсона [9], Старка [10]. 
В работе Синая и Ханина [8] доказан следующий результат. Пусть $f \in$ $C^{2+\nu}\left(S^{1}\right), \nu>0, f^{\prime}(x)>0, x \in S^{1}$, и число вращения $\rho$ является диофантовым с показателем $\delta$, т.е. существует константа $c(\rho)$, такая, что

$$
\left|\rho-\frac{p}{q}\right| \geqslant \frac{c(\rho)}{q^{2+\delta}} \quad \text { для любого } \frac{p}{q} \in \mathbb{Q}
$$

и $\delta<\nu$. Тогда сопряжение $\varphi$ принадлежит $C^{1+\nu-\delta}$. Отметим, что условие $f \in C^{2+\nu}$ неулучшаемо, поскольку для множества чисел вращения полной лебеговой меры существуют примеры диффеоморфизмов класса $C^{2}$, для которых приведение существенно сингулярно.

Тесно связана с вопросом о гладкости сопряжения $\varphi$ и проблема существования абсолютно непрерывной инвариантной меры для $T$. Хорошо известно (см. [12]), что всякий гомеоморфизм окружности с иррациональным числом вращения является строго эргодическим, т.е. обладает единственной, с точностью до нормировки, инвариантной мерой. При этом инвариантная мера $\mu$ для $T$ является абсолютно непрерывной относительно меры Лебега тогда и только тогда, когда сопряжение $\varphi$ задается абсолютно непрерывной функцией. Это соображение было впервые использовано В. И. Арнольдом [2]. Таким образом, для $C^{2+\nu}$-гладких диффеоморфизмов в случае типичных чисел вращения инвариантная мера абсолютно непрерывна относительно меры Лебега.

Оказывается, что при наличии одной особенности типа излома ситуация меняется на диаметрально противоположную. А именно, инвариантная мера всегда сингулярна относительно меры Лебега.

Целью нашей работы является доказательство следующего результата:

Теорема 1. Пусть функиия $f(x)$, определяющал гомеоморфизм $T$, удовлетворяет условиям (a)-(d) и число врачения $\rho=\rho(T)$ иррачионально. Тогда инвариантная мера н сингулярна относительно меры Лебега $\lambda$, m. е. существует измеримое подмножество $A$ окружности, $A \subset S^{1}$, такое, ито $\mu(A)=1, \lambda(A)=0$.

Авторы признательны Я. Г. Синаю за полезные обсуждения.

\section{§2. Необходимые определения и факты}

Рассмотрим сохраняющий ориентацию гомеоморфизм окружности $T$, определяемый функцией $f(x), x \in \mathbb{R}^{1}$ :

$$
T x=\{f(x)\}, \quad x \in S^{1} .
$$

Пусть число вращения $\rho$, отвечающее $T$, иррационально. Возьмем произвольную точку $x_{0} \in S^{1}$ и рассмотрим траекторию этой точки под действием $T$, т.е. множество точек $\left\{x_{i}=T^{i} x_{0}, i \in \mathbb{Z}^{1}\right\}$. Всюду в дальнейшем $T^{i}$ обозначает $i$-ю итерацию отображения $T$. Согласно классической теореме Пуанкаре (см. $[11,12])$, порядок точек вдоль траектории будет в точности таким же, как и для линейного поворота окружности $T_{\rho}$, т. е. для последовательности $\left\{\bar{x}_{i}=\left\{x_{0}+\rho i\right\}, i \in \mathbb{Z}^{1}\right\}$. Это важное свойство позволяет определить 
систему естественных разбиений окружности, связанных с разложением $\rho$ в непрерывную дробь.

Пусть $\rho=\left[k_{1}, k_{2}, \ldots, k_{n}, \ldots\right]$ и $p_{n} / q_{n}=\left[k_{1}, k_{2}, \ldots, k_{n}\right], n \geqslant 1$. Числа $p_{n} / q_{n}$ называются подходящими дробями для $\rho$. Числитель $p_{n}$ и знаменатель $q_{n}$ удовлетворяют рекуррентным соотношениям

$$
\begin{aligned}
& p_{n}=k_{n} p_{n-1}+p_{n-2}, \quad n \geqslant 2, \quad p_{0}=0, p_{1}=1, \\
& q_{n}=k_{n} q_{n-1}+q_{n-2}, \quad n \geqslant 2, \quad q_{0}=1, q_{1}=k_{1} \text {. }
\end{aligned}
$$

Для произвольной точки $x_{0} \in S^{1}$ обозначим через $\Delta_{0}^{(n)}\left(x_{0}\right)$ замкнутый интервал, концами которого служат точки $x_{0}$ и $x_{q_{n}}=T^{q_{n}} x_{0}$. Заметим, что при нечетном $n$ точка $x_{q_{n}}$ лежит слева от $x_{0}$, а при четном $n$ - справа. Через $\Delta_{i}^{(n)}\left(x_{0}\right)$ обозначим итерации интервала $\Delta_{0}^{(n)}\left(x_{0}\right)$ :

$$
\Delta_{i}^{(n)}\left(x_{0}\right)=T^{i} \Delta_{0}^{(n)}\left(x_{0}\right)
$$

Лемма 1. Рассмотрим произвольную точку $x_{0} \in S^{1}$. Отрезок траектории этой точки $\left\{x_{i}, 0 \leqslant i<q_{n}+q_{n-1}\right\}$ разбивает окружность на непересекаюшиеся (за исключением концевых точек) отрезки $\Delta_{i}^{(n-1)}\left(x_{0}\right)$, $0 \leqslant i<q_{n}, \Delta_{j}^{(n)}\left(x_{0}\right), 0 \leqslant j<q_{n-1}$.

Лемма 1 хорошо известна и носит по существу арифметический характер, поскольку структура разбиения определяется порядком точек траектории $\left\{x_{i}, 0 \leqslant i<q_{n}+q_{n-1}\right\}$, который совпадает с порядком точек для линейного поворота $T_{\rho}$. Возникающее разбиение обозначим через $\xi_{n}\left(x_{0}\right)$ и назовем динамическим разбиением $n$-го порядка. Ниже мы кратко опишем структуру динамических разбиений. При переходе от $\xi_{n}\left(x_{0}\right) \mathrm{K} \xi_{n-1}\left(x_{0}\right)$ происходит следующее. Все отрезки $n$-го ранга сохраняются, а каждый из отрезков $\Delta_{i}^{(n-1)}\left(x_{0}\right), 0 \leqslant i<q_{n}$, разбивается на $k_{n+1}+1$ отрезков (см. рис. 1$)$ :

$$
\Delta_{i}^{(n-1)}\left(x_{0}\right)=\Delta_{i}^{(n+1)}\left(x_{0}\right) \cup \bigcup_{s=0}^{k_{n+1}-1} \Delta_{i+q_{n-1}+s q_{n}}^{(n)}\left(x_{0}\right) \text {. }
$$

Динамические разбиения $\xi_{m}\left(x_{0}\right)$ измельчаются с ростом $m, \xi_{m+1}\left(x_{0}\right)>$ $\xi_{m}\left(x_{0}\right)$ в том смысле, что каждый элемент предыдущего разбиения состоит из объединения целого числа элементов следующего разбиения.

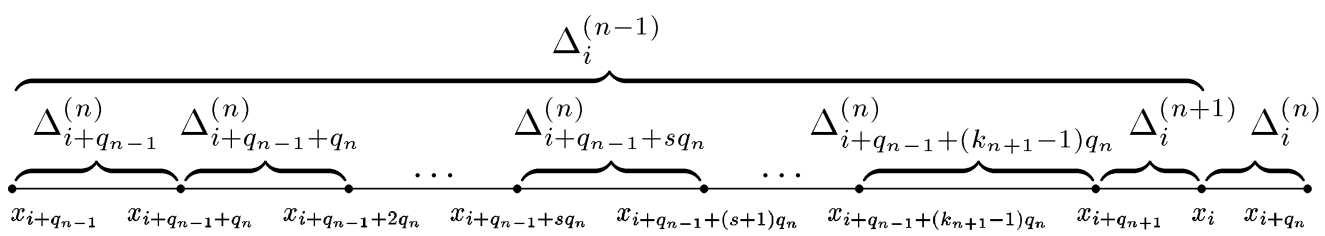

Рис. 1

ЛЕмМА ДАНжУА. Пусть существуют конечные односторонние производнье $f^{\prime}\left(x_{c}-0\right), f^{\prime}\left(x_{c}+0\right)>0, f \in C^{1}\left(\left[x_{c}, x_{c}+1\right]\right) u \operatorname{var}_{\left[x_{c}, x_{c}+1\right]} \ln f^{\prime}=\bar{\theta}<\infty$. Положим

$$
\theta=\bar{\theta}+\left|\ln f^{\prime}\left(x_{c}-0\right)-\ln f^{\prime}\left(x_{c}+0\right)\right|=\bar{\theta}+2|\ln c| .
$$


Тогда для любого $x_{0}$, такого, ито $x_{i} \neq x_{c}, i=0,1,2, \ldots$, справедливо неравенство

$$
e^{-\theta} \leqslant \prod_{s=0}^{q_{k}-1} f^{\prime}\left(x_{i}\right) \leqslant e^{\theta} .
$$

Лемма Данжуа доказывается так же, как аналогичное утверждение в работе [13].

Из леммы Данжуа следует, что отрезки, составляющие динамическое разбиение $\xi_{n}\left(x_{0}\right)$, имеют экспоненциально малую длину. Положим

$$
\lambda=\left(1+e^{-\theta}\right)^{1 / 2} .
$$

СлЕдСТВиЕ 1. Пусть $\Delta^{(n)}-$ произвольньй элемент динамического разбиения $\xi_{n}\left(x_{0}\right)$. Тогда

$$
\left|\Delta^{(n)}\right| \leqslant \text { const } \lambda^{n}
$$

где $|\cdot|$ означает длину отрезка.

ОПРЕДЕЛЕНИЕ 1. Два гомеоморфизма окружности $T_{1}$ и $T_{2}$ называются топологически эквивалентными, если существует такой гомеоморфизм $\varphi: S^{1} \rightarrow$ $S^{1}$, что $\varphi\left(T_{1} x\right)=T_{2} \varphi(x)$ для любого $x \in S^{1}$. При этом гомеоморфизм $\varphi$ называется сопрягающим или сопряжением. Из следствия 1 легко вытекает теорема Данжуа.

ТЕОРемА ДАНЖУА. Пусть гомеоморфизм $T$ с иррачиональным числом вращения $\rho$ удовлетворяет условиям леммь Данжуа. Тогда T топологически эквивалентен линейному повороту $T_{\rho}$.

Из следствия 1 вытекает, что траектория любой точки плотно заполняет окружность. Отсюда следует, что сопряжение существует и определяется единственным образом с точностью до поворота. С другой стороны, хорошо известно (см. $[2,12])$, что если $\mu$ - нормированная инвариантная мера, то в качестве $\varphi$ можно взять отображение

$$
\varphi(x)=\mu\left(\left[x_{0}, x\right]\right), \quad x \in S^{1},
$$

где $x_{0}$ - произвольная точка окружности.

В дальнейшем важную роль играет понятие двойного отношения (crossratio).

ОПРЕДЕЛЕНИЕ 2. Двойным отношением четверки $\left(z_{1}, z_{2}, z_{3}, z_{4}\right), z_{1}<z_{2}<$ $z_{3}<z_{4}$, называется число

$$
\operatorname{Cr}\left(z_{1}, z_{2}, z_{3}, z_{4}\right)=\frac{\left(z_{2}-z_{1}\right)\left(z_{4}-z_{3}\right)}{\left(z_{3}-z_{1}\right)\left(z_{4}-z_{2}\right)} .
$$

Нам необходима следующая лемма.

Лемма 2 (см. [14]). Пусть $f \in C^{2+\nu}([a, b]), \nu>0$. Тогда существует такая постоянная $C$, ито

$$
\left|\frac{\operatorname{Cr}\left(f\left(z_{1}\right), f\left(z_{2}\right), f\left(z_{3}\right), f\left(z_{4}\right)\right)}{\operatorname{Cr}\left(z_{1}, z_{2}, z_{3}, z_{4}\right)}-1\right| \leqslant C\left(\left(z_{4}-z_{1}\right)^{1+\nu}\right)
$$

для любой четверки чисел $\left(z_{1}, z_{2}, z_{3}, z_{4}\right), a \leqslant z_{1}<z_{2}<z_{3}<z_{4} \leqslant b$. 


\section{§3. Формулировка основных лемм и доказательство теоремы 1}

В этом параграфе мы приведем основные леммы и, используя их, докажем теорему 1.

Пусть $z_{i} \in S^{1}, i=1,2,3,4, z_{1}<z_{2}<z_{3}<z_{4}$. Введем обозначения

$$
\alpha=z_{2}-z_{1}, \quad \beta=z_{3}-z_{2}, \quad \gamma=z_{4}-z_{3} .
$$

ЛЕмма 3. Пусть в точке $x=x_{0}$ сопряжение $\varphi(x)$ имеет положительную производную $\varphi^{\prime}\left(x_{0}\right)=p_{0}>0, x_{0} \in S^{1}$, и для некоторой константь $R>1$ выполнены следуюшие условия:

(a) $\alpha / R \leqslant \beta \leqslant R \alpha, \alpha / R \leqslant \gamma \leqslant R \alpha$;

(b) $\max _{1 \leqslant i \leqslant 4}\left|z_{i}-x_{0}\right| \leqslant R \alpha$.

Тогда для любого $\varepsilon>0$ суиествует $\delta=\delta(\varepsilon)>0$, такое, ито если все $z_{i}$, $1 \leqslant i \leqslant 4$, лежат в $\left(x_{0}-\delta, x_{0}+\delta\right)$, то справедливо неравенство

$$
\left|\frac{\operatorname{Cr}\left(\varphi\left(z_{1}\right), \varphi\left(z_{2}\right), \varphi\left(z_{3}\right), \varphi\left(z_{4}\right)\right)}{\operatorname{Cr}\left(z_{1}, z_{2}, z_{3}, z_{4}\right)}-1\right| \leqslant C_{1} \varepsilon,
$$

где константа $C_{1}$ зависит лишь от $R$ u $p_{0}$, и не зависит от $\varepsilon$.

Пусть $x_{c} \in\left[z_{1}, z_{2}\right]$. Введем также следующие обозначения:

$$
\begin{gathered}
\tau=x_{c}-z_{1}, \quad \alpha^{\prime}=T z_{2}-T z_{1}, \quad \beta^{\prime}=T z_{3}-T z_{2}, \quad \gamma^{\prime}=T z_{4}-T z_{3}, \\
\operatorname{Cr}(\alpha, \beta, \gamma)=\frac{\alpha}{\alpha+\beta} \frac{\gamma}{\beta+\gamma}, \quad \operatorname{Cr}\left(\alpha^{\prime}, \beta^{\prime}, \gamma^{\prime}\right)=\frac{\alpha^{\prime}}{\alpha^{\prime}+\beta^{\prime}} \frac{\gamma^{\prime}}{\beta^{\prime}+\gamma^{\prime}}, \\
F(z)=\frac{(1+\xi)\left(\left(c^{2}-1\right) z+1\right)}{\left(c^{2}-1\right) z+1+\xi}, \quad \text { где } z=\frac{\tau}{\alpha}, \xi=\frac{\beta}{\alpha} .
\end{gathered}
$$

ЛЕмма 4. Пусть функиия $f$, определяющая гомеоморфизм $T$, принадлежит классу $C^{2}\left(S^{1} \backslash\left\{x_{c}\right\}\right), f^{\prime}(x) \geqslant$ const $>0$ и существует константа $R>1$, такая, что выполняются неравенства

$$
\alpha / R<\beta<R \alpha, \quad \alpha / R<\gamma<R \alpha .
$$

Тогда существует константа $C_{2}=C_{2}(f, R)$, такал, что

$$
\left|\frac{\operatorname{Cr}\left(\alpha^{\prime}, \beta^{\prime}, \gamma^{\prime}\right)}{\operatorname{Cr}(\alpha, \beta, \gamma)}-F(z)\right| \leqslant C_{2}(\alpha+\beta+\gamma) .
$$

ОПРЕдЕлЕниЕ 3. Скажем, что тройка отрезков $\left(\left[z_{1}, z_{2}\right],\left[z_{2}, z_{3}\right],\left[z_{3}, z_{4}\right]\right)$ накрывает особую точку $x_{c}$, если $x_{c} \in\left(z_{1}, z_{4}\right)$. Тройка отрезков накрывает особую точку «правильным» образом с константой $M, 0<M \leqslant 1$, если

1) $x_{c} \in\left(z_{1}, z_{2}\right]$;

2) $\left(x_{c}-z_{1}\right) /\left(z_{2}-z_{1}\right) \geqslant M$.

Лемма 5. Пусть определяюшая функиия $f(x)$ гомеоморфизма $T$ удовлетворяет условиям теореми 1. Тогда для любого $x_{0} \in S^{1}$ и для любого $\delta>0$ суиествует $N\left(\delta, x_{0}\right)>0$, такое, что для всех $n>N\left(\delta, x_{0}\right)$ найдется тройка отрезков $\left[z_{s}, z_{s+1}\right] \subset\left(x_{0}-\delta, x_{0}+\delta\right), s=1,2,3$, со следуюшими свойствами: 
1) $\left|T^{j}\left[z_{1}, z_{4}\right]\right| \leqslant$ const $\lambda^{n}, 0 \leqslant j<q_{n}$, аде $\lambda=\left(1+e^{-\theta}\right)^{-1 / 2}, a|\cdot|$ обозначает длину отрезка;

2) $\sum_{j=0}^{q_{n}-1}\left|T^{j}\left[z_{1}, z_{4}\right]\right| \leqslant 2$

3) отрезки $\left[z_{s}, z_{s+1}\right] u T^{q_{n}}\left[z_{s}, z_{s+1}\right], s=1,2,3$, удовлетворяют условиям а) $и$ b) леммы 3 сконстантой $R=e^{3 \theta}+e^{\theta}+1$;

4) троика отрезков $T^{j}\left[z_{1}, z_{2}\right], T^{j}\left[z_{2}, z_{3}\right], T^{j}\left[z_{3}, z_{4}\right], 0 \leqslant j \leqslant q_{n}$, накрывает особую точку $x=x_{c}$ ровно один раз; при этом накрытие является «правильньм» с константой $M=1$.

ЛЕмма 6. Предположим, что определяюшая функиия $f(x)$ гомеоморфизма $T$ удовлетворяет условиям теоремь 1 и отрезки $\left[z_{s}, z_{s+1}\right], s=1,2,3$, удовлетворяют утверждениям 1)-4) леммь 5. Тогда для достаточно больших $n$ имеют место следуюшие неравенства:

(a)

$$
\begin{aligned}
& \frac{\operatorname{Cr}\left(T^{q_{n}} z_{1}, T^{q_{n}} z_{2}, T^{q_{n}} z_{3}, T^{q_{n}} z_{4}\right)}{\operatorname{Cr}\left(z_{1}, z_{2}, z_{3}, z_{4}\right)}>\text { const }>1 \quad \text { npu } c>1, \\
& \frac{\operatorname{Cr}\left(T^{q_{n}} z_{1}, T^{q_{n}} z_{2}, T^{q_{n}} z_{3}, T^{q_{n}} z_{4}\right)}{\operatorname{Cr}\left(z_{1}, z_{2}, z_{3}, z_{4}\right)}<\mathrm{const}<1 \quad \text { npu } c<1,
\end{aligned}
$$

где константы зависят лишь от функции $f$.

ДоКАЗАТЕЛЬСТво ТЕОРЕмЫ 1 . Поскольку инвариантная мера $\mu$ не имеет атомов и $\varphi(x)$ задается монотонной функцией,

$$
\varphi(x)=\int_{0}^{x} d \mu(x)
$$

где $\mu(x)$ - инвариантная мера, нам достаточно показать, что для почти всех $x$ по мере Лебега $\varphi^{\prime}(x)=0$. Производная $\varphi^{\prime}(x)$ существует в силу монотонности функции $\varphi$ для почти всех $x$ по мере Лебега. Мы покажем, что $\varphi^{\prime}(x)=0$ всюду, где производная определена.

Предположим, что $\varphi^{\prime}\left(x_{0}\right)=\theta>0, x_{0} \in S^{1}$. Для определенности предположим, что величина излома $c$ больше 1 . Фиксируем $\varepsilon>0$. Возьмем тройку отрезков $\left(\left[z_{1}, z_{2}\right],\left[z_{2}, z_{3}\right],\left[z_{3}, z_{4}\right]\right),\left[z_{s}, z_{s+1}\right] \subset\left(x_{0}-\delta, x_{0}+\delta\right), s=1,2,3$, удовлетворяющих условиям леммы 5. Тогда эти отрезки и отрезки $\left[T^{q_{n}} z_{s}, T^{q_{n}} z_{s+1}\right]$, $s=1,2,3$, удовлетворяют условиям леммы 3 . Из леммы 3 следует, что

$$
\begin{gathered}
\left|\frac{\operatorname{Cr}\left(\varphi\left(z_{1}\right), \varphi\left(z_{2}\right), \varphi\left(z_{3}\right), \varphi\left(z_{4}\right)\right)}{\operatorname{Cr}\left(z_{1}, z_{2}, z_{3}, z_{4}\right)}-1\right|<C_{1} \varepsilon, \\
\left|\frac{\operatorname{Cr}\left(\varphi\left(T^{q_{n}} z_{1}\right), \varphi\left(T^{q_{n}} z_{2}\right), \varphi\left(T^{q_{n}} z_{3}\right), \varphi\left(T^{q_{n}} z_{4}\right)\right)}{\operatorname{Cr}\left(T^{q_{n}} z_{1}, T^{q_{n}} z_{2}, T^{q_{n}} z_{3}, T^{q_{n}} z_{4}\right)}-1\right|<C_{1} \varepsilon .
\end{gathered}
$$

Поскольку $\varphi$ осуществляет сопряжение с линейным поворотом, нетрудно видеть, что

$$
\operatorname{Cr}\left(\varphi\left(T^{q_{n}} z_{1}\right), \varphi\left(T^{q_{n}} z_{2}\right), \varphi\left(T^{q_{n}} z_{3}\right), \varphi\left(T^{q_{n}} z_{4}\right)\right)=\operatorname{Cr}\left(\varphi\left(z_{1}\right), \varphi\left(z_{2}\right), \varphi\left(z_{3}\right), \varphi\left(z_{4}\right)\right) .
$$

Из формул (3)-(5) непосредственно вытекает, что

$$
\left|\frac{\operatorname{Cr}\left(T^{q_{n}} z_{1}, T^{q_{n}} z_{2}, T^{q_{n}} z_{3}, T^{q_{n}} z_{4}\right)}{\operatorname{Cr}\left(z_{1}, z_{2}, z_{3}, z_{4}\right)}-1\right|<C_{3} \varepsilon
$$


где константа $C_{3}>0$ не зависит от $\varepsilon$ и $n$. С другой стороны, из леммы 6 следует, что для достаточно больших $n$

$$
\frac{\operatorname{Cr}\left(T^{q_{n}} z_{1}, T^{q_{n}} z_{2}, T^{q_{n}} z_{3}, T^{q_{n}} z_{4}\right)}{\operatorname{Cr}\left(z_{1}, z_{2}, z_{3}, z_{4}\right)}>\text { const }>1 .
$$

При достаточно малых $\varepsilon$ последнее неравенство и неравенство (6) одновременно не могут выполняться. Это противоречие доказывает теорему 1.

\section{§4. Доказательства лемм 3-6}

ДоКАЗАТЕЛЬСТвО ЛЕМмЫ 3 . Пусть существует $\varphi^{\prime}\left(x_{0}\right)=p_{0}>0$. По определению производной для любого $\varepsilon>0$ существует $\delta=\delta\left(x_{0}, \varepsilon\right)>0$, такое, что для всех $x \in\left(x_{0}-\delta, x_{0}+\delta\right)$ имеют место неравенства

$$
p_{0}-\varepsilon<\frac{\varphi(x)-\varphi\left(x_{0}\right)}{x-x_{0}}<p_{0}+\varepsilon .
$$

Возьмем точки $z_{i} \in\left(x_{0}-\delta, x_{0}+\delta\right)$, удовлетворяющие условиям (a), (b) леммы 3. Для определенности предположим, что $z_{i}<x_{0}, i=1,2,3,4$. В остальных случаях лемма 3 доказывается аналогично. При $x=z_{i}, i=$ $1,2,3,4$, из (7) вытекает, что

$$
\left(p_{0}-\varepsilon\right)\left(x_{0}-z_{i}\right)<\varphi\left(x_{0}\right)-\varphi\left(z_{i}\right)<\left(p_{0}+\varepsilon\right)\left(x_{0}-z_{i}\right) .
$$

Отсюда легко можно вывести следующие неравенства:

$$
\begin{aligned}
& p_{0}-\varepsilon \frac{\left(x_{0}-z_{2}\right)+\left(x_{0}-z_{1}\right)}{z_{2}-z_{1}} \leqslant \frac{\varphi\left(z_{2}\right)-\varphi\left(z_{1}\right)}{z_{2}-z_{1}} \leqslant p_{0}+\varepsilon \frac{\left(x_{0}-z_{2}\right)+\left(x_{0}-z_{1}\right)}{z_{2}-z_{1}}, \\
& p_{0}-\varepsilon \frac{\left(x_{0}-z_{3}\right)+\left(x_{0}-z_{1}\right)}{z_{3}-z_{1}} \leqslant \frac{\varphi\left(z_{3}\right)-\varphi\left(z_{1}\right)}{z_{3}-z_{1}} \leqslant p_{0}+\varepsilon \frac{\left(x_{0}-z_{3}\right)+\left(x_{0}-z_{1}\right)}{z_{3}-z_{1}}, \\
& p_{0}-\varepsilon \frac{\left(x_{0}-z_{4}\right)+\left(x_{0}-z_{2}\right)}{z_{4}-z_{2}} \leqslant \frac{\varphi\left(z_{4}\right)-\varphi\left(z_{2}\right)}{z_{4}-z_{2}} \leqslant p_{0}+\varepsilon \frac{\left(x_{0}-z_{4}\right)+\left(x_{0}-z_{2}\right)}{z_{4}-z_{2}}, \\
& p_{0}-\varepsilon \frac{\left(x_{0}-z_{4}\right)+\left(x_{0}-z_{3}\right)}{z_{4}-z_{3}} \leqslant \frac{\varphi\left(z_{4}\right)-\varphi\left(z_{3}\right)}{z_{4}-z_{3}} \leqslant p_{0}+\varepsilon \frac{\left(x_{0}-z_{4}\right)+\left(x_{0}-z_{3}\right)}{z_{4}-z_{3}} .
\end{aligned}
$$

Из условий (a) и (b) леммы 3 вытекает, что

$$
\max _{1 \leqslant i \leqslant 4} \max \left\{\frac{x_{0}-z_{i}}{z_{2}-z_{1}}, \frac{x_{0}-z_{i}}{z_{3}-z_{1}}, \frac{x_{0}-z_{i}}{z_{4}-z_{2}}, \frac{x_{0}-z_{i}}{z_{4}-z_{3}}\right\} \leqslant K_{1},
$$

где константа $K_{1}>0$ зависит лишь от $R$ и не зависит от $\varepsilon$. Очевидно, что

$$
\begin{aligned}
& \frac{\operatorname{Cr}\left(\varphi\left(z_{1}\right), \varphi\left(z_{2}\right), \varphi\left(z_{3}\right), \varphi\left(z_{4}\right)\right)}{\operatorname{Cr}\left(z_{1}, z_{2}, z_{3}, z_{4}\right)} \\
& \quad=\frac{\varphi\left(z_{2}\right)-\varphi\left(z_{1}\right)}{z_{2}-z_{1}} \frac{\varphi\left(z_{4}\right)-\varphi\left(z_{3}\right)}{z_{4}-z_{3}} \frac{z_{3}-z_{1}}{\varphi\left(z_{3}\right)-\varphi\left(z_{1}\right)} \frac{z_{4}-z_{2}}{\varphi\left(z_{4}\right)-\varphi\left(z_{2}\right)} .
\end{aligned}
$$

Отсюда, используя (9)-(13), нетрудно получить утверждение леммы 3. Лемма 3 доказана. 
ДокАЗАТЕЛЬСТво ЛЕммы 4. По условию $x_{c} \in\left[z_{1}, z_{2}\right]$. Следовательно, отрезок $\left[z_{2}, z_{4}\right]$ не содержит особую точку $x_{c}$. Очевидно, что

$$
\begin{aligned}
\frac{\gamma^{\prime}}{\beta^{\prime}+\gamma^{\prime}} & =\frac{T z_{4}-T z_{3}}{T z_{4}-T z_{2}}=\frac{f^{\prime}\left(x_{c}+0\right) \gamma+O\left(\gamma^{2}\right)}{f^{\prime}\left(x_{c}+0\right)(\beta+\gamma)+O\left((\beta+\gamma)^{2}\right)} \\
& =\frac{\gamma}{\beta+\gamma} \frac{1+O(\gamma)}{1+O(\beta+\gamma)}=\frac{\gamma}{\beta+\gamma}(1+O(\beta+\gamma)), \\
\frac{\alpha^{\prime}}{\alpha^{\prime}+\beta^{\prime}} & =\frac{f^{\prime}\left(x_{c}-0\right) \tau+f^{\prime}\left(x_{c}+0\right)(\alpha-\tau)+O\left(\alpha^{2}\right)}{f^{\prime}\left(x_{c}-0\right) \tau+f^{\prime}\left(x_{c}+0\right)(\alpha+\beta-\tau)+O\left((\alpha+\beta)^{2}\right)} \\
& =\frac{\left(c^{2}-1\right) z+1+O(\alpha)}{\left(c^{2}-1\right) z+1+\xi+O(\alpha+\beta)}=\frac{\left(c^{2}-1\right) z+1}{\left(c^{2}-1\right) z+1+\xi}(1+O(\alpha+\beta)) .
\end{aligned}
$$

Из последнего соотношения вытекает, что

$$
\frac{\alpha^{\prime}}{\alpha^{\prime}+\beta^{\prime}} \frac{\alpha+\beta}{\alpha}=\frac{(1+\xi)\left(\left(c^{2}-1\right) z+1\right)}{\left(c^{2}-1\right) z+1+\xi}(1+O(\alpha+\beta)) .
$$

Отсюда и из равенства (14) легко получить неравенство (2). Лемма 4 доказана.

ДоКАЗАТЕЛЬСТво ЛЕмМЫ 5 . Пусть $\varphi^{\prime}\left(x_{0}\right)=p_{0}>0$ и разложение числа вращения в непрерывную дробь имеет вид $\rho=\left[k_{1}, k_{2}, \ldots, k_{n}, \ldots\right]$. Рассмотрим динамическое разбиение $\xi_{n}\left(x_{0}\right)$. Элементами разбиения $\xi_{n}\left(x_{0}\right)$ являются отрезки $\Delta_{i}^{(n-1)}\left(x_{0}\right), 0 \leqslant i<q_{n}, \Delta_{j}^{(n)}\left(x_{0}\right), 0 \leqslant i<q_{n-1}$.

Возможны два случая:

$$
x_{c} \in \Delta_{i_{0}}^{(n-1)}\left(x_{0}\right), \quad 0 \leqslant i_{0}<q_{n}, \quad \text { или } \quad x_{c} \in \Delta_{i_{0}}^{(n)}\left(x_{0}\right), \quad 0 \leqslant i_{0}<q_{n-1} .
$$

Обозначим через $\bar{x}_{c}$ прообраз точки $x_{c}$, лежащий внутри отрезка $\Delta_{0}^{(n)}\left(x_{0}\right)$ или $\Delta_{0}^{(n-1)}\left(x_{0}\right)$. Введем следующие обозначения:

$$
z_{1}=T^{-q_{n-1}}\left(\bar{x}_{c}\right), \quad z_{2}=\bar{x}_{c}, \quad z_{3}=\frac{\bar{x}_{c}+T^{q_{n-1}}\left(\bar{x}_{c}\right)}{2}, \quad z_{4}=T^{q_{n-1}}\left(\bar{x}_{c}\right) .
$$

Рассмотрим систему отрезков $\left[z_{s}, z_{s+1}\right], s=1,2,3$, и их образы $T^{j}\left[z_{s}, z_{s+1}\right]$, $0 \leqslant j<q_{n}$. Заметим, что $T^{i_{0}} z_{2}=x_{c}, 0 \leqslant i_{0}<q_{n}$. Поэтому образ отрезка $\left[z_{2}, z_{4}\right]$ никогда не содержит точку $x_{c}$ внутри себя. Действительно, отрезок $\left[z_{2}, z_{4}\right]$ является образующим для разбиения $\xi_{n}\left(\bar{x}_{c}\right),\left[z_{2}, z_{4}\right]=\Delta_{0}^{(n-1)}\left(\bar{x}_{c}\right)$. Кроме того, $T^{i_{0}}\left[z_{2}, z_{4}\right], 0 \leqslant i_{0}<q_{n}$, содержит точку $x_{c}$ на границе элемента разбиения. Отрезок $\left[z_{1}, z_{2}\right]$ также является образующим для разбиения $\xi_{n}\left(T^{-q_{n-1}} \bar{x}_{c}\right),\left[z_{1}, z_{2}\right]=\Delta_{0}^{(n-1)}\left(T^{-q_{n-1}} \bar{x}_{c}\right)$. Поскольку $T^{i_{0}} z_{2}=x_{c}$, образы $T^{j}\left[z_{1}, z_{2}\right], 0 \leqslant j<q_{n}$, отрезка $\left[z_{1}, z_{2}\right]$ также не содержат точку $x_{c}$ внутри элементов разбиения. Таким образом, система отрезков $T^{j}\left(\left[z_{1}, z_{2}\right],\left[z_{2}, z_{3}\right]\right.$, $\left.\left[z_{3}, z_{4}\right]\right), 0 \leqslant j<q_{n}$, покрывает особую точку ровно один раз при $j=i_{0}$. Очевидно, что покрытие является правильным с константой $M=1$. Таким образом, условие 4) леммы 5 выполнено. Условия 1) и 2) выполняются очевидным образом. Действительно, $T^{j}\left(\left[z_{1}, z_{4}\right]\right)$ является объединением двух отрез- 
ков $(n-1)$-го ранга. Таким образом, используя следствие 1 к лемме Данжуа, получаем

$$
\left|T^{j}\left(\left[z_{1}, z_{4}\right]\right)\right| \leqslant \text { const } \lambda^{n}, \quad 0 \leqslant j \leqslant q_{n} .
$$

Кроме того,

$$
\sum_{j=0}^{q_{n}-1}\left|T^{j}\left(\left[z_{1}, z_{4}\right]\right)\right|=\sum_{j=0}^{q_{n}-1}\left|T^{j}\left(\left[z_{1}, z_{2}\right]\right)\right|+\sum_{j=0}^{q_{n}-1}\left|T^{j}\left(\left[z_{2}, z_{4}\right]\right)\right| \leqslant 2 .
$$

Нам осталось проверить выполнение условий (a) и (b) леммы 3. Из леммы Данжуа очевидным образом следует, что

$$
\begin{aligned}
e^{-\theta}\left|\left[z_{1}, z_{2}\right]\right| \leqslant\left|\left[z_{2}, z_{4}\right]\right| & =\left|T^{q_{n-1}}\left[z_{1}, z_{2}\right]\right| \leqslant e^{\theta}\left|\left[z_{1}, z_{2}\right]\right|, \\
e^{-\theta}\left|T^{q_{n}}\left[z_{1}, z_{2}\right]\right| \leqslant\left|T^{q_{n}}\left[z_{2}, z_{4}\right]\right| & =\left|T^{q_{n-1}} T^{q_{n}}\left[z_{1}, z_{2}\right]\right| \leqslant e^{\theta}\left|T^{q_{n}}\left[z_{1}, z_{2}\right]\right| .
\end{aligned}
$$

Кроме того, $\left|\left[z_{1}, z_{2}\right]\right|=\left|\left[z_{3}, z_{4}\right]\right|$ и

$$
e^{-2 \theta} \leqslant\left|T^{q_{n}}\left[z_{2}, z_{3}\right]\right| /\left|T^{q_{n}}\left[z_{3}, z_{4}\right]\right| \leqslant e^{2 \theta} .
$$

Таким образом, условие (а) леммы 3 с константой $R=e^{\theta}\left(1+e^{2 \theta}\right)$ выполнено для $\left[z_{s}, z_{s+1}\right], s=1,2,3$, и $T^{q_{n}}\left(\left[z_{s}, z_{s+1}\right]\right), s=1,2,3$. Проверим теперь условие (b) леммы 3. Нетрудно убедится, что отрезок $\left[z_{1}, z_{4}\right]$ содержит внутри себя точку $x_{0}$. Таким образом,

$$
\max _{1 \leqslant s \leqslant 4}\left|z_{s}-x_{0}\right| \leqslant\left|\left[z_{1}, z_{4}\right]\right| \leqslant\left(1+e^{\theta}\right)\left|\left[z_{1}, z_{2}\right]\right| .
$$

В случае отрезков $T^{q_{n}}\left(\left[z_{s}, z_{s+1}\right]\right), s=1,2,3$, рассмотрим два случая. Если $\bar{x}_{c} \in \Delta_{i}^{(n-1)}\left(x_{0}\right)$, то $T^{q_{n}}\left[z_{1}, z_{4}\right]$ содержит точку $x_{0}$, поскольку точка $T^{q_{n}} z_{4}$ лежит правее $z_{2}$ (см. рис. 2). Тогда

$$
\max _{1 \leqslant s \leqslant 4}\left|T^{q_{n}} z_{s}-x_{0}\right| \leqslant\left(1+e^{\theta}\right)\left|T^{q_{n}}\left[z_{1}, z_{2}\right]\right| \text {. }
$$

$\longrightarrow T^{q_{n}} z_{1} \quad z_{1}=T^{-q_{n-1}} z_{2} \quad x_{0} \quad z_{2}=\bar{x}_{c} \quad T^{q_{n}} z_{4} \quad \bullet$

Рис. 2

$T^{q_{n}} z_{1} \quad z_{1}=T^{-q_{n-1}} \bar{x}_{c} \quad x_{q_{n}} \quad z_{2}=\bar{x}_{c} \quad T^{q_{n}} z_{4} \quad x_{0} \quad z_{4}=T^{q_{n-1}} z_{2} \quad x_{q_{n-1}}$

Рис. 3

Во втором случае, когда $\bar{x}_{c} \in \Delta_{i_{0}}^{(n)}\left(x_{0}\right)$, отрезок $T^{q_{n}}\left[z_{1}, z_{4}\right]$ может не содержать точку $x_{0}$. Однако и в этом случае точка $T^{q_{n}} z_{4}$ расположена правее (см. рис. 3). Поэтому

$$
\begin{aligned}
\max _{1 \leqslant s \leqslant 4}\left|T^{q_{n}} z_{s}-x_{0}\right| & \leqslant\left|T^{q_{n}}\left[z_{1}, z_{4}\right]\right|+\left|\left[z_{2}, z_{4}\right]\right| \leqslant\left(1+e^{\theta}\right)\left|T^{q_{n}}\left[z_{1}, z_{2}\right]\right|+e^{\theta}\left|\left[z_{1}, z_{2}\right]\right| \\
& \leqslant\left(1+e^{\theta}\right)\left|T^{q_{n}}\left[z_{1}, z_{2}\right]\right|+e^{2 \theta}\left|T^{q_{n}}\left[z_{1}, z_{2}\right]\right| \\
& =\left(e^{2 \theta}+e^{\theta}+1\right)\left|T^{q_{n}}\left[z_{1}, z_{2}\right]\right| .
\end{aligned}
$$


Окончательно получаем, что условия (a), (b) леммы 3 выполнены для отрезков $\left[z_{s}, z_{s+1}\right], T^{q_{n}}\left(\left[z_{s}, z_{s+1}\right]\right), s=1,2,3$, с константой $R=e^{3 \theta}+e^{\theta}+1$. Лемма 5 доказана.

ДОКАЗАТЕЛЬСТВО ЛЕМмЫ 6. ПредПоложим для определенности, что $c>1$. В случае $c<1$ лемма 6 доказывается аналогично. Пусть отрезки $\left[z_{s}, z_{s+1}\right]$, $s=1,2,3$, удовлетворяют условиям леммы 5 и тройка отрезков $\left(T^{i_{0}}\left[z_{1}, z_{2}\right]\right.$, $\left.T^{i_{0}}\left[z_{2}, z_{3}\right], T^{i_{0}}\left[z_{3}, z_{4}\right]\right), 0 \leqslant i_{0}<q_{n}$, накрывает особую точку. Очевидно, что

$$
\begin{aligned}
I= & \frac{\operatorname{Cr}\left(T^{q_{n}} z_{1}, T^{q_{n}} z_{2}, T^{q_{n}} z_{3}, T^{q_{n}} z_{4}\right)}{\operatorname{Cr}\left(z_{1}, z_{2}, z_{3}, z_{4}\right)} \\
= & \prod_{\substack{i=0, i \neq i_{0}}}^{q_{n}-1} \frac{\operatorname{Cr}\left(T^{i+1} z_{1}, T^{i+1} z_{2}, T^{i+1} z_{3}, T^{i+1} z_{4}\right)}{\operatorname{Cr}\left(T^{i} z_{1}, T^{i} z_{2}, T^{i} z_{3}, T^{i} z_{4}\right)} \\
& \times \frac{\operatorname{Cr}\left(T^{i_{0}+1} z_{1}, T^{i_{0}+1} z_{2}, T^{i_{0}+1} z_{3}, T^{i_{0}+1} z_{4}\right)}{\operatorname{Cr}\left(T^{i_{0}} z_{1}, T^{i_{0}} z_{2}, T^{i_{0}} z_{3}, T^{i_{0}} z_{4}\right)} .
\end{aligned}
$$

По предположению среди отрезков $T^{i}\left[z_{1}, z_{4}\right], i=0,1, \ldots, q_{n}$, только отрезок $T^{i_{0}}\left[z_{1}, z_{4}\right]$ содержит особую точку. Используя лемму 2 и условие 1$)$ леммы 5 , получаем

$$
\begin{aligned}
& \prod_{\substack{i=0, i \neq i_{0}}}^{q_{n}-1} \frac{\operatorname{Cr}\left(T^{i+1} z_{1}, T^{i+1} z_{2}, T^{i+1} z_{3}, T^{i+1} z_{4}\right)}{\operatorname{Cr}\left(T^{i} z_{1}, T^{i} z_{2}, T^{i} z_{3}, T^{i} z_{4}\right)}=\prod_{\substack{i=0, i \neq i_{0}}}^{q_{n}-1}\left(1+O\left(\left|\left[T^{i} z_{1}, T^{i} z_{4}\right]\right|^{1+\nu}\right)\right) \\
= & \exp \left\{\sum_{\substack{i=0, i \neq i_{0}}}^{q_{n}-1} \ln \left(1+O\left(\left|\left[T^{i} z_{1}, T^{i} z_{4}\right]\right|^{1+\nu}\right)\right)\right\}=1+O\left(\lambda^{n \nu}\right) .
\end{aligned}
$$

Теперь оценим второй сомножитель в (23). Используя леммы 4 и 5 , получаем неравенство

$$
\left|\frac{\operatorname{Cr}\left(T^{i_{0}+1} z_{1}, T^{i_{0}+1} z_{2}, T^{i_{0}+1} z_{3}, T^{i_{0}+1} z_{4}\right)}{\operatorname{Cr}\left(T^{i_{0}} z_{1}, T^{i_{0}} z_{2}, T^{i_{0}} z_{3}, T^{i_{0}} z_{4}\right)}-F(x)\right| \leqslant C_{2} \lambda^{n},
$$

где

$$
F(x)=\frac{(1+\xi)\left(\left(c^{2}-1\right) x+1\right)}{\left(c^{2}-1\right) x+1+\xi}, \quad \xi=\frac{\left|T^{i_{0}} z_{3}-T^{i_{0}} z_{2}\right|}{\left|T^{i_{0}} z_{2}-T^{i_{0}} z_{1}\right|}, \quad x=\frac{\left|x_{c}-T^{i_{0}} z_{1}\right|}{\left|T^{i_{0}} z_{2}-T^{i_{0}} z_{1}\right|} .
$$

Легко видеть, что функция $F(x), 0 \leqslant x \leqslant 1$, является строго возрастающей (при $c>1$ ), причем $F(0)=1$. По предположению тройка отрезков ([T $\left.\left.T^{i_{0}} z_{1}, T^{i_{0}} z_{2}\right],\left[T^{i_{0}} z_{2}, T^{i_{0}} z_{3}\right],\left[T^{i_{0}} z_{3}, T^{i_{0}} z_{4}\right]\right)$ «правильным» образом накрывает особую точку с константой $M=1$. Отсюда следует, что

$$
\frac{\operatorname{Cr}\left(T^{i_{0}+1} z_{1}, T^{i_{0}+1} z_{2}, T^{i_{0}+1} z_{3}, T^{i_{0}+1} z_{4}\right)}{\operatorname{Cr}\left(T^{i_{0}} z_{1}, T^{i_{0}} z_{2}, T^{i_{0}} z_{3}, T^{i_{0}} z_{4}\right)} \geqslant F(1)-C_{2} \lambda^{n} .
$$

Поскольку $F(1)=c^{2}(1+\xi) /\left(c^{2}+\xi\right)$ и $\xi \geqslant 1 / R=\left(e^{3 \theta}+e^{\theta}+1\right)^{-1}$, получаем $F(1) \geqslant c^{2}(R+1) /\left(R c^{2}+1\right)=1+\left(c^{2}-1\right) /\left(R c^{2}+1\right)>1$. Отсюда и из $(23)-(26)$ легко вытекает утверждение леммы 6 . 


\section{ЛИТЕРАТУРА}

1. Denjoy $A$. Sur les courbes definies par les equations differentielles a la surface du tore. J. Math. Pures Appl., 11, 333-375 (1932).

2. Арнольд В. И. Малые знаменатели І. Об отображениях окружности на себя. Изв. АН СССР, сер. мат., 25, вып. 1, 21-86 (1961).

3. Moser J. A rapidly convergent iteration method, Part II, Ann. Scuola Norm. Sup. Pisa Cl. Sci. (3), 20, 499-535 (1966).

4. Herman $M$. Sur la conjugaison différentiable des diféomorphisims du cercle a des rotations, Publ. Math. IHES, 49, 5-233 (1979).

5. Herman $M$. Résultats récents sur la conjugaison différentiable. Proc. Intern. Cong. Math., Helsinki, Vol. 2, 1978, pp. 811-820.

6. Yocсоz J. C. Conjugaison différentiable des diféomorphisims du cercle dont la mombre de rotation vérifie une condition diophantienne. Ann. Sci. École Norm. Sup. (4), 17, 333-359 (1984).

7. Khanin K. M., Sinai Ya. G. A new proof of M. Herman's theorem. Comm. Math. Phys., 112, 89-101 (1987).

8. Синай Я. Г., Ханин К. М. Гладкость сопряжений диффеоморфизмов окружности с поворотами. УМН, 44, вып. 1 (265), 57-81 (1989).

9. Katznelson $Y$, Ornstein D. The differentiability of the conjugation of certain diffeomorphisms of the circle. Ergodic Theory Dynam. Systems, 9, No. 4, 643-680 (1989).

10. Stark J. Smooth conjugacy and renormalization for diffeomorphisms of the circle. Nonlinearity, 1, 541-575 (1988).

11. Poincaré $H$. Memoire sur les courbes definie par une equation differentieble I-IV. J. Math. Pures Appl., 1881-1886. [Имеется русский перевод: О кривых, определяемых дифференциальными уравнениями, Гостехиздат, М.-Л., 1947.]

12. Корнфельд И. П., Синай Я. Г., Фомин С. В. Эргодическая теория. Наука, М., 1980.

13. Khanin K. M., Vul E. B. Circle homeomorphisms with weak discontinuties. Adv. Sov. Math., Vol. 3, 57-98 (1991).

14. Khanin K. M. Universal estimates for critical circle mappings. Chaos, 1, 181 (1991).

Самаркандский госуниверситет, механико-математический факультет Институт теоретической физики им. Л. Д. Ландау и Хериот-Ватт Университет (Эдинбург)
Поступило в редакцию 19 февраля 1998 г. 